\title{
EDITORIAL
}

\section{Medical treatment for pregnant women: balancing risks and benefits}

\section{Journal of Perinatology (2009) 29, 589-590; doi:10.1038/jp.2009.68}

Pregnant women are prone to medical diseases requiring treatment similar to non-pregnant individuals. However, unlike for nonpregnant women and men, medical therapies may not have been studied in the setting of pregnancy and there are concerns regarding the effect of such therapies on the developing fetus. ${ }^{1}$ Thus, when the obstetrician, serving as the primary physician for pregnant women, facilitates diagnosis and treatment of conditions in pregnancy, the risks and benefits of treatment, suboptimal treatment, and no treatment all must be carefully considered and discussed with the patient.

In this issue of the Journal of Perinatology, Wataganara et al. ${ }^{2}$ describe a case of severe achalasia during pregnancy successfully treated with esophagoscopic injection of botulinum toxin A. The patient presented with progressive dysphagia, severe malnutrition and fetal growth restriction at 33 weeks' gestation. Her condition mandated total parenteral nutrition. After counseling, a treatment course with botulinum toxin A intrasphincteric injection was adopted. Her condition improved and she delivered a healthy baby 3 weeks later.

Achalasia is a neurodegenerative esophageal disease that not only involves the myenteric plexus of the esophagus, but also the vagal trunks and dorsal vagal nucleus. ${ }^{3}$ In pregnancy it has been associated with maternal malnutrition and maternal death, as well as preterm delivery, fetal growth restriction and fetal demise. ${ }^{4,5}$ Although the use of botulinum toxin $\mathrm{A}$ as part of a treatment modality may seem broadly uncontroversial for this rare disorder at least in the non-pregnant state, pregnancy often has a way of altering the risk/benefit calculation for the standard medical therapies. This alteration in the calculus of therapeutic options, although often appropriate, can also be draconian and categorical, and thus exclude the pregnant patient of beneficial diagnostic as well as medical and surgical interventions.

One need only consider the hesitancy regarding use of routine and appropriate imaging in pregnancy for fear of fetal radiation exposure, ${ }^{6}$ as well as the controversies over the use of chemotherapy in pregnancy, ${ }^{7}$ and to a greater extent, radiation therapy. ${ }^{8}$ This hesitancy, fueled by both a reasonable concern for fetal harm, as well as fear of malpractice exposure from the medico-legal industry, can often degrade nuanced and innovative medical decision making.

The concern with botulinum toxin A, as the authors note, is that it is a neurotoxin, little studied in pregnancy, and with at least the potential for fetal/neonatal neuromuscular blockade. Given the limited evidence, the authors approached this dilemma in a thoughtful and methodical manner. The range of available therapies was assessed. The best therapy for the patient's medical condition - irrespective of pregnancy - determined (often a very simple question can help our medicine and surgery colleagues maintain the proper perspective: 'what would you do if this patient wasn't pregnant?'). Next, the risks of that 'optimal' therapy to the pregnancy were evaluated objectively using all available evidence. In the end, there is always a fine balance that must be attained between maternal and fetal risk and benefit. It is crucial that the patient remains a full partner in this complex decision making, and that her threshold for risk either to herself or to the unborn baby inform the final decision.

We can all appreciate that medical imaging should be performed as indicated in pregnancy, that fetal radiation exposure risks should be balanced with optimizing maternal care, that even chemotherapy and radiation therapy are appropriate options in the well-counseled pregnant patient, ${ }^{6}$ that not every drug is a Thalidomide story, and that the history of Bendectin is an unfortunate chapter. We can only hope that with a thoughtful adjudication of risks and benefits in a well-counseled patient, the diagnostic, medical and surgical horizons for pregnant patients will continue to expand.

\section{Conflict of interest}

The authors declare no conflict of interest.

YY El-Sayed ${ }^{1}$ and AB Caughey ${ }^{2}$

${ }^{1}$ Department of Obstetrics and Gynecology, Stanford University, Stanford, CA, USA and ${ }^{2}$ Department of Obstetrics and Gynecology, University of California, San Francisco, San Francisco, CA, USA E-mail: abcmd@berkeley.edu 


\section{References}

1 Lyerly AD, Little M0, Faden RR. Pregnancy and clinical research. Hastings Cent Rep 2008; 38(6): inside back cover.

2 Wataganara T, Leelakusolvong S, Sunsaneevithayakul P, Vantanasiri C. Treatment of severe achalasia during pregnancy with esophagoscopic injection of botulinum toxin A: a case report. J Perinatol 2009; this issue.

3 Nguyen NQ, Holloway RH. Recent developments in esophageal motor disorders. Curr Opin Gastroenterol 2005; 21: 478-484.

4 Ohno J, Kawai M, Shibata Y, Arii Y. Esophageal achalasia in pregnancy. Am J Perinatol 2000; 17: 53-55
5 Fassina G, Osculati A. Achalasia and sudden death: a case report. Forensic Sci Int 1995; 75: 133-137.

6 Brent RL. Saving lives and changing family histories: appropriate counseling of pregnant women and men and women of reproductive age, concerning the risk of diagnostic radiation exposures during and before pregnancy. Am J Obstet Gynecol 2009; 1: 4-24.

7 Cardonick E, Iacobucci A. Use of chemotherapy during human pregnancy. Lancet Oncol 2004; 5(5): 283-291.

8 Kal HB, Struikmans H. Radiotherpay during pregnancy: fact and fiction. Lancet Oncol 2005; 6(5): 328-333. 